\title{
Correction to: Is 14-Days a Sensible Quarantine Length for COVID-19? Examinations of Some Associated Issues with a Case Study of COVID-19 Incubation Times
}

Yasin Khadem Charvadeh ${ }^{1}$. Grace Y. Yi ${ }^{1,2} \cdot$ Yuan Bian ${ }^{1}$. Wenqing He ${ }^{1}$ (1)

Published online: 21 September 2021

(c) International Chinese Statistical Association 2021

\section{Correction to: Statistics in Biosciences}

\section{https://doi.org/10.1007/s12561-021-09320-8}

The original version of this article unfortunately contained error in Acknowledgement section.

Therefore, the correct and complete section is given here:

Acknowledgements The research is partially supported by funding from the Discovery Grant Program and the Emerging Infectious Disease Modeling Program of the Natural Sciences and Engineering Research Council of Canada (NSERC). Yi is Canada Research Chair in Data Science (Tier 1). Her research was undertaken, in part, thanks to funding from the Canada Research Chairs Program.

The original article has been corrected.

The original article can be found online at https://doi.org/10.1007/s12561-021-09320-8.

Wenqing $\mathrm{He}$

whe@stats.uwo.ca

1 Department of Statistical and Actuarial Sciences, University of Western Ontario, London, ON, Canada

2 Department of Computer Science, University of Western Ontario, London, ON, Canada 